\title{
IRREDUCIBLE LIE ALGEBRAS OF INFINITE TYPE
}

\author{
ROBERT LEE WILSON ${ }^{1}$
}

Abstract. Let $V$ be a finite dimensional vector space over an algebraically closed field of characteristic $\neq 2,3,5$. It is shown that if $L \subseteq \mathrm{gl}(V)$ is an irreducible Lie algebra of infinite type then either $L=\operatorname{gl}(V), L=\operatorname{sl}(V), \operatorname{dim} V=2 r \geqq 4$ and $L=\operatorname{sp}(V), \operatorname{dim} V=2 r \geqq 4$ and $L=\operatorname{csp}(V)$, or there exists $A \in L$ such that ad $A \neq 0=(\operatorname{ad} A)^{2}$. As a corollary we obtain E. Cartan's classification of the irreducible Lie algebras of infinite type over $C$.

Let $L$ be a Lie algebra of linear transformations of a vector space $V$. For each nonnegative integer $n$ the $n$th Cartan prolongation, $L_{n}$, is defined inductively by $L_{0}=L$ and

$$
L_{n}=\left\{\phi \in \operatorname{Hom}\left(V, L_{n-1}\right) \mid y(x \phi)=x(y \phi) \text { for all } x, y \in V\right\}
$$

for $n \geqq 1$. If $L_{n} \neq(0)$ for all $n \geqq 0$ then $L$ is said to be of infinite type. The main result of this paper is:

Theorem 1. Let $V$ be a finite dimensional vector space over an algebraically closed field of characteristic $\neq 2,3,5$. If $L$ is an irreducible Lie algebra of infinite type then either $L=\operatorname{gl}(V), L=\operatorname{sl}(V), \operatorname{dim} V=2 r$ $\geqq 4$ and $L=\operatorname{sp}(V), \operatorname{dim} V=2 r \geqq 4$ and $L=\operatorname{csp}(V)$, or there exists an $A \in L$ such that ad $A \neq 0=(\operatorname{ad} A)^{2}$.

Now it is easily seen (as in [7]) that if $(\operatorname{ad} A)^{2}=0$ then $A$ belongs to the radical of the Killing form. If $\Phi=\boldsymbol{C}$ and $L$ is reductive this implies that ad $A=0$. Furthermore, it is known (Theorem 1 of [4]) that an irreducible Lie algebra over $\boldsymbol{C}$ is of infinite type if and only if $L_{2} \neq(0)$. Thus Theorem 1 implies the following theorem of E. Cartan [1]:

THeOREM 2. Let $V$ be a finite dimensional vector space over $\mathbf{C}$ and let $L$ be an irreducible Lie algebra of linear transformations of $V$ such that $L_{2} \neq(0)$. Then either $L=\operatorname{gl}(V), L=\operatorname{sl}(V), \operatorname{dim} V=2 r \geqq 4$ and $L=\operatorname{sp}(V)$, or $\operatorname{dim} V=2 r \geqq 4$ and $L=\operatorname{csp}(V)$.

Theorem 2, which is important in the study of primitive pseudogroups and infinite Lie algebras of Cartan type, has been proved by several authors $([1],[2],[3],[5],[9])$. These proofs have involved considerable use of the classification and representation theory of

Received by the editors August 17, 1970.

AMS 1970 subject classifications. Primary 17B20; Secondary 17B65.

Key words and phrases. Cartan prolongation, infinite Lie algebra of Cartan type.

1 The author has received partial support from a Sloan Foundation grant. 
semisimple Lie algebras over $C$ and hence cannot be generalized to fields of prime characteristic. In the proof presented here we use more elementary techniques which are valid over algebraically closed fields of characteristic $\neq 2,3,5$.

We will consider the following three conditions on a Lie algebra $L$ of linear transformations of a vector space $V$ :

Condition A. There exists $A \in L$ with rank $A=1$ and $A^{2} \neq 0$.

Condition B. Either $\operatorname{dim} V=2$ or there exist $A, B \in L$ with rank $A$ $=\operatorname{rank} B=1, \operatorname{ker} A=\operatorname{ker} B$, and $V A \neq V B$.

Condition C. There exists $A \in L$ with ad $A \neq 0=(\operatorname{ad} A)^{2}$.

By Condition $\sim \mathrm{X}$ we will mean the negation of Condition $\mathrm{X}$. Theorem 1 is clearly a consequence of the following two lemmas.

LEMMA 1. If $\Phi$ is an algebraically closed field of arbitrary characteristic, $V$ is a finite dimensional vector space over $\Phi$, and $L \subseteq \operatorname{gl}(V)$ is a Lie algebra of infinite type then $L$ contains a rank one transformation.

LEMмa 2. Let $V$ be a finite dimensional vector space over a field $\Phi$ of characteristic $\neq 2,3,5$. Let $L$ be an irreducible Lie algebra of linear transformations of $V$. Assume that $L$ contains a rank one transformation. Then:

(i) If Condition A holds $L=\operatorname{gl}(V)$.

(ii) If Conditions $\sim \mathrm{A}, \mathrm{B}$, and $\sim \mathrm{C}$ hold then $L=\mathrm{sl}(V)$.

(iii) If Conditions $\sim \mathrm{A}, \sim \mathrm{B}$, and $\sim \mathrm{C}$ hold then $\operatorname{dim} V=2 r \geqq 4$ and $L=\operatorname{sp}(V)$ or $L=\operatorname{csp}(V)$.

It is shown in [2] that if $\Phi=C$ Lemma 1 is a consequence of Hilbert's Nullstellensatz. The proof given there is in fact independent of the assumption $\Phi=C$ and could be used to prove our Lemma 1 . We will give a somewhat more elementary proof here. Part (i) of Lemma 2 is proved in [6] for the case $\Phi=C$.

In the proof of Lemma 1 we will need:

Lемма 3. Let $\Phi$ be an algebraically closed field of arbitrary characteristic. Let $V$ and $W$ be finite dimensional vector spaces over $\Phi$ with $2 \leqq \operatorname{dim} V \leqq \operatorname{dim} W$. Let $T$ be a subspace of $\operatorname{Hom}(V, W)$ such that $\operatorname{dim} T \geqq \operatorname{dim} W$. Then there exist $\phi \in T$ and $v \in V$ such that $\phi \neq 0$, $v \neq 0$, and $v \phi=0$.

Proof. Suppose $T \subseteq \operatorname{Hom}(V, W)$ is a counterexample to the lemma such that $\operatorname{dim} W$ is minimal among all counterexamples. As $T$ is a counterexample we have, for any nonzero $v \in V, \operatorname{dim} W \geqq \operatorname{dim} v T$ $=\operatorname{dim} T \geqq \operatorname{dim} W$. Thus for any $w \in W$ there is a unique $\phi \in T$ such that $v \phi=w$. Then if $v_{1}, v_{2}$ are linearly independent elements of $V$ we can find a basis $\left\{\psi_{1}, \cdots, \psi_{n}\right\}$ of $T$ such that 


$$
\begin{aligned}
& v_{2} \psi_{i}=v_{1} \psi_{i+1} \text { for all } 1 \leqq i \leqq n-1, \text { and } \\
& v_{2} \psi_{n}=\sum_{i=1}^{n} v_{1} \psi_{i} a_{i} \text { where the } a_{i} \in \Phi
\end{aligned}
$$

(For if linearly independent elements $\psi_{1}, \cdots, \psi_{j}$ satisfying (1) have been found for some $1 \leqq j \leqq n-1$ then the minimality of $\operatorname{dim} W \mathrm{im}$ plies that $v_{2} \psi_{j} \notin\left\langle v_{1} \psi_{1}, \cdots, v_{1} \psi_{j}\right\rangle$. Thus there exists a unique $\psi_{j+1} \in T$ such that $\psi_{1}, \cdots, \psi_{j+1}$ are linearly independent and satisfy (1). Proceeding by induction on $j$ gives the result.) Now there exists $\lambda \in \Phi$ such that $\lambda^{n}-\sum_{i=0}^{n-1} \lambda^{i} a_{i+1}=0$. Setting $b_{k}=\lambda^{n-k}$ $-\sum_{i=0}^{n-k-1} \lambda^{2} a_{k+i+1}$ for $1 \leqq k \leqq n-1$ we see that $\psi=\psi_{n}+\sum_{i=1}^{n-1} \psi_{i} b_{i} \in T$ and $\left(v_{2}-\lambda v_{1}\right) \psi=0$. This contradicts the choice of $T$ and proves the lemma.

Proof of Lemma 1. For $\phi \in L_{i}$ and $j \leqq i$ define

$$
\operatorname{im}_{j}(\phi)=\left\langle v_{j}\left(\cdots\left(v_{1} \phi\right) \cdots\right) \mid v_{1}, \cdots, v_{j} \in V\right\rangle .
$$

Now if $x \in \operatorname{ker} \phi$ and $y \in V$ then $0=y(x \phi)=x(y \phi)$ so we have ker $\phi$ $\subseteq \operatorname{ker}(y \phi)$. Thus if $\psi \in \operatorname{im}_{j}(\phi)$ we have ker $\psi \supseteq \operatorname{ker} \phi$. Furthermore if $d_{i}=\min \left\{\right.$ rank $\left.\phi \mid 0 \neq \phi \in L_{i}\right\}$ we have $d_{0} \leqq d_{1} \leqq \cdots \leqq \operatorname{dim} V$. Hence there is some integer $N$ such that $d_{N}=d_{N+i}$ for all $i \geqq 0$. Now if $i \geqq j \geqq 0$ and if $\phi \in L_{N+i}$ satisfies rank $\phi=d_{N}$ then for $0 \neq \psi \in \operatorname{im}_{j}(\phi)$ we have $\operatorname{ker} \psi \supseteq \operatorname{ker} \phi$ and rank $\psi \geqq d_{N}=\operatorname{rank} \phi$. Thus $\operatorname{ker} \psi=\operatorname{ker} \phi$. Thus $\operatorname{im}_{j}(\phi) \subseteq \operatorname{Hom}\left(V / \operatorname{ker} \phi, \operatorname{im}_{j+1}(\phi)\right)$ and $0 \neq \psi \in \operatorname{im}_{j}(\phi)$ implies rank $\psi=d_{N}=\operatorname{dim}(V /$ ker $\phi)$. Thus if $d_{N} \geqq 2$, Lemma 3 shows that $\operatorname{dim} \operatorname{im}_{j}(\phi) \leqq \operatorname{dim} \operatorname{im}_{j+1}(\phi)-1$. Hence $\operatorname{dim} L_{N-1} \geqq \operatorname{dim} \operatorname{im}_{i+1}(\phi) \geqq$ $\operatorname{dim} \operatorname{im}_{1}(\phi)+i \geqq i$ for all $i \geqq 0$. Since $L_{N-1}$ is finite dimensional this is impossible. Hence $1=d_{N}=d_{0}$, proving the lemma.

Proof of Lemma 2. Let $n=\operatorname{dim} V$. If $\left\{x_{1}, \cdots, x_{n}\right\}$ and $\left\{y_{1}, \cdots, y_{n}\right\}$ are bases for $V$ we define elements $E_{i j}$ and $F_{i j} \in \operatorname{gl}(V)$ for $1 \leqq i, j \leqq n$ by $x_{k} E_{i j}=\delta_{k i} x_{j}$ and $y_{k} F_{i j}=\delta_{k i} y_{j}$. If $2 k \leqq n$ we define $\operatorname{sp}\left(x_{1}, \cdots, x_{2 k}\right)$ to be the Lie algebra of all $A \in \operatorname{gl}(V)$ such that $V A \subseteq\left\langle x_{1}, \cdots, x_{2 k}\right\rangle, x_{r} A=0$ for all $r>2 k$, and $A$ is skew with respect to the skew-symmetric bilinear form defined by $\left(x_{2 i+1}, x_{j}\right)=\delta_{j, 2 i+2}$, $\left(x_{2 i+2}, x_{j}\right)=-\delta_{j, 2 i+1}$, and $\left(x_{r}, x_{j}\right)=0$ for all $0 \leqq i \leqq k-1,1 \leqq j \leqq n$, $2 k<r \leqq n$.

We will presently verify the following statements about an irreducible Lie algebra $L$ of linear transformations of $V$ :

(a) If $1 \leqq k<n$ and $E_{1 i} \in L$ for all $1 \leqq i \leqq k$ then there is a basis $\left\{y_{1}, \cdots, y_{n}\right\}$ of $V$ such that $F_{1 i} \in L$ for all $1 \leqq i \leqq k+1$.

(b) If $1 \leqq k<n, E_{1 i} \in L$ for all $1 \leqq i \leqq n$, and $E_{i 1} \in L$ for all $1 \leqq i \leqq k$ then there is a basis $\left\{y_{1}, \cdots, y_{n}\right\}$ of $V$ such that $F_{1 i} \in L$ for all $1 \leqq i \leqq n$ and $F_{i 1} \in L$ for all $1 \leqq i \leqq k+1$. 
(c) If $2 \leqq k \leqq m \leqq n, E_{1 i} \in L$ for all $2 \leqq i \leqq m, E_{i 1} \in L$ for all $2 \leqq i<k$, and $L$ satisfies Condition $\sim \mathrm{C}$ then there is a basis $\left\{y_{1}, \cdots, y_{n}\right\}$ of $V$ such that $F_{1 i} \in L$ for all $2 \leqq i \leqq m$ and $F_{i 1} \in L$ for all $2 \leqq i \leqq k$.

(d) If $3 \leqq k<n$ and $E_{1 i}, E_{i 1} \in L$ for all $2 \leqq i \leqq k$ then there is a basis $\left\{y_{1}, \cdots, y_{n}\right\}$ of $V$ such that $F_{1 i} \in L$ for all $2 \leqq i \leqq k+1$ and $F_{i 1} \in L$ for all $2 \leqq i \leqq k$.

(e) If $\operatorname{sp}\left(x_{1}, \cdots, x_{2 k}\right) \subseteq L$ where $\operatorname{dim} V>2 k \geqq 2$ and $L$ satisfies Conditions $\sim \mathrm{B}$ and $\sim \mathrm{C}$ then $\operatorname{dim} V \geqq 2 k+2$ and there is a basis $\left\{y_{1}, \cdots, y_{n}\right\}$ of $V$ such that $\operatorname{sp}\left(y_{1}, \cdots, y_{2 k+2}\right) \subseteq L$.

(f) If $\operatorname{sp}(V) \subseteq L$ and $L$ satisfies Condition $\sim \mathrm{B}$ then $L=\operatorname{sp}(V)$ or $L=\operatorname{csp}(V)$.

Lemma 2 follows immediately from statements (a)-(f). For if Condition A holds we may choose a basis $\left\{x_{1}, \cdots, x_{n}\right\}$ for $V$ such that $E_{11} \in L$. Then by (a), (b), and induction on $k$ we see that $L$ $=\operatorname{gl}(V)$, proving (i). If Conditions $\sim \mathrm{A}, \mathrm{B}$, and $\sim \mathrm{C}$ hold we may choose a basis $\left\{x_{1}, \cdots, x_{n}\right\}$ of $V$ such that $E_{12} \in L$ and if $n \geqq 3$ we may also arrange that $E_{13} \in L$. Then using (c), (d), and induction on $k$ we see that $L=\operatorname{sl}(V)$, proving (ii). Finally if Conditions $\sim \mathrm{A}, \sim \mathrm{B}$, and $\sim \mathrm{C}$ hold we may choose a basis $\left\{x_{1}, \cdots, x_{n}\right\}$ of $V$ such that $E_{12} \in L$. By (c) we may assume that $\operatorname{sp}\left(x_{1}, x_{2}\right) \subseteq L$. Then by (e) and induction on $k$ we have $\operatorname{dim} V=2 r \geqq 4$ and $\operatorname{sp}(V) \subseteq L$. Then (f) proves (iii).

We now verify (a)-(f). Throughout we will let $A=\sum E_{i j} a_{i j}$ where the $a_{i j} \in \Phi$.

(a): As $\left\langle x_{1}, \cdots, x_{k}\right\rangle$ is not an invariant subspace there exists $A \in L$ such that $a_{i j} \neq 0$ for some $1 \leqq i \leqq k<j \leqq n$. We may assume that $i=1$ (replacing $A$ by $A\left(\operatorname{ad} E_{1 i}\right)$ if $\left.i \neq 1\right)$ and that $a_{r j}=0$ whenever $r>1$ or $j=1$ (replacing $A$ by $\left.\left(A\left(\operatorname{ad} E_{11}\right)-A\right)\left(\operatorname{ad} E_{11}\right) / 2\right)$. Letting $\left\{y_{1}, \cdots, y_{n}\right\}$ be any basis for $V$ satisfying $y_{i}=x_{i}$ for $1 \leqq i \leqq k, y_{k+1}$ $=x_{1} A$, and $y_{j} \in\left\langle x_{k+1}, \cdots, x_{n}\right\rangle$ for $\left.j\right\rangle k+1$ gives the result.

The proof of (b) is similar to that of (a).

(c): First assume that ad $E_{1 k}=0$. Then for every $A \in L$ we have $0=A\left(\operatorname{ad} E_{1 k}\right)=\sum_{i}\left(E_{i k} a_{i 1}-E_{1 i} a_{k i}\right)$. Hence $a_{k i}=0$ for all $i \neq k$ so $\left\langle x_{k}\right\rangle$ is an invariant subspace, contradicting the irreducibility of $L$. Hence ad $E_{1 k} \neq 0$ so by Condition $\sim \mathrm{C}$ we have $\left(\operatorname{ad} E_{1 k}\right)^{2} \neq 0$. Since $A\left(\operatorname{ad} E_{1 k}\right)^{2}=E_{1 k}\left(-2 a_{k 1}\right)$ we have $E_{1 k} \in L\left(\operatorname{ad} E_{1 k}\right)^{2}$. Since $E_{1 k}^{2}=0$ we have $\left(\operatorname{ad} E_{1 k}\right)^{3}=0$. Thus Lemma V.8.2 of [8] shows that there exists $A \in L$ such that $A\left(\operatorname{ad} E_{1 k}\right)^{2}=2 E_{1 k}$ and $A\left(\operatorname{ad} E_{1 k}\right)(\operatorname{ad} A)=-2 A$. Now

$$
A\left(\operatorname{ad} E_{1 k}\right)(\operatorname{ad} A)=\sum_{i, j} E_{i j}\left(2 a_{k j} a_{i 1}-\delta_{i 1} \sum_{r} a_{k r} a_{r j}-\delta_{j k} \sum_{r} a_{i r} a_{r 1}\right) .
$$


Thus we have $a_{k 1}=-1$ and

$$
a_{i j}=-a_{i 1} a_{k j}+\left(\delta_{i 1} \sum_{r} a_{k r} a_{r j}+\delta_{j k} \sum_{r} a_{i r} a_{r 1}\right) / 2 .
$$

Setting $i=j=1$ gives $0=\sum_{r} a_{k r} a_{r 1}$. From this, using (2) to substitute for $a_{r j}$ in $\sum_{r} a_{k r} a_{r j}$, we conclude that $\sum_{r} a_{k r} a_{r j}=0$ for all $1 \leqq j \leqq n$ and similarly that $\sum_{r} a_{i r} a_{r 1}=0$ for all $1 \leqq i \leqq n$. Thus $a_{i j}=-a_{i 1} a_{k j}$ and $\sum_{r} a_{i r} a_{r j}=0$ for $1 \leqq i, j \leqq n$. Now set $y_{1}=x_{k} A, y_{k}=x_{k}$, and $y_{j}=x_{j}$ $+a_{j 1} x_{k}$ for $j \neq 1, k$. Then $F_{k 1}=A \in L, F_{1 k}=-E_{1 k} \in L$, and $F_{1 i}=-E_{1 k} a_{i 1}$ $-E_{1 i} \in L$ for $2 \leqq i \leqq m, i \neq k$. Finally for $2 \leqq i<k$ we have $F_{i 1}=$ $-E_{i 1}\left(\operatorname{ad} F_{1 k}\right)\left(\operatorname{ad} F_{k 1}\right)-\left(F_{11}-F_{k k}\right) a_{k i} \in L$ as required.

(d): As in (a) we may find a basis $\left\{y_{1}, \cdots, y_{n}\right\}$ of $V$ such that $F_{1 i}, F_{i 1} \in L$ for $1 \leqq i \leqq k$ and $A=\sum F_{i j} b_{i j} \in L$ where $b_{1 j}=\delta_{j, k+1}$. Then

$$
F_{1, k+1}=A\left(\operatorname{ad} F_{21}\right)\left(\operatorname{ad} F_{12}\right)\left(\operatorname{ad} F_{31}\right)\left(\operatorname{ad} F_{13}\right)+\left(F_{11}-F_{33}\right) b_{22} \in L
$$

as required.

(e): It is well known (for example [8, p. 67] where we take $x_{2 i+1}$ $=v_{i+1}$ and $x_{2 i+2}=v_{r+i+1}$ for $\left.0 \leqq i \leqq r-1\right)$ that $\operatorname{sp}\left(x_{1}, \cdots, x_{2 r}\right)$ is spanned by the following elements and their transposes: $E_{2 i+1,2 i+1}$ $-E_{2 i+2,2 i+2}, E_{2 i+1,2 i+2}, E_{2 i+1,2 j+1}-E_{2 j+2,2 i+2}$, and $E_{2 i+1,2 j+2}+E_{2 j+1,2 i+2}$ for $0 \leqq i \neq j \leqq r-1$. Thus it is easily checked that $\operatorname{sp}\left(x_{1}, \cdots, x_{2 k+2}\right)$ is generated by $\operatorname{sp}\left(x_{1}, \cdots, x_{2 k}\right), E_{1,2 k+1}-E_{2 k+2,2}$, and $E_{2 k+1,2 k+2}$.

We first show that for some basis $\left\{y_{1}, \cdots, y_{n}\right\}$ of $V$ we have $\operatorname{sp}\left(y_{1}, \cdots, y_{2 k}\right) \subseteq L$ and $A=F_{1,2 k+1}+\sum_{i>2 k} F_{i 2} b_{i 2} \in L$ where the $b_{i 2}$ $\in \Phi$. As $\left\langle x_{1}, \cdots, x_{2 k}\right\rangle$ is not an invariant subspace there exists $A \in L$ such that $a_{i j} \neq 0$ for some $1 \leqq i \leqq 2 k<j \leqq n$. We may assume that $i=1$ (replacing $A$ by $A\left(\operatorname{ad} E_{12}\right)$ if $i=2$, by $A\left(\operatorname{ad}\left(E_{1,2 r+1}-E_{2 r+2,2}\right)\right.$ ) if $i=2 r+1$, and by $A\left(\operatorname{ad}\left(E_{1,2 r+2}+E_{2 r+1,2}\right)\right)$ if $\left.i=2 r+2\right)$, that $a_{r s}=0$ unless $r=1, s>2$ or $r>2, s=2$ (replacing $A$ by $A\left(\operatorname{ad} E_{21}\right)\left(\operatorname{ad} E_{12}\right)$ $\left.-\left(E_{11}-E_{22}\right)\left(a_{11}-a_{22}\right)-E_{12}\left(2 a_{12}\right)\right)$, and that $a_{1 s}=a_{82}=0$ for $s \leqq 2 k$ (replacing $A$ by

$A-\sum_{i=1}^{k-1} A\left(\left(\operatorname{ad} E_{2 i+2,2 i+1}\right)\left(\operatorname{ad} E_{2 i+1,2 i+2}\right)+\left(\operatorname{ad} E_{2 i+1,2 i+2}\right)\left(\operatorname{ad} E_{2 i+2,2 i+1}\right)\right)$.

Then letting $\left\{y_{1}, \cdots, y_{n}\right\}$ be any basis for $V$ satisfying $x_{i}=y_{i}$ for $1 \leqq i \leqq 2 k, y_{2 k+1}=x_{1} A$, and $y_{j} \in\left\langle x_{2 k+1}, \cdots, x_{n}\right\rangle$ for $j>2 k+1$ gives the result.

Now assume that $\operatorname{sp}\left(x_{1}, \cdots, x_{2 k}\right) \subseteq L$ and that

$$
A=E_{1,2 k+1}+\sum_{i>2 k} E_{i 2} a_{i 2} \in L \text {. }
$$


We will show that for some basis $\left\{y_{1}, \cdots, y_{n}\right\}$ we have $\operatorname{sp}\left(y_{1}, \cdots, y_{2 k}\right)$ $\subseteq L$ and $F_{1,2 k+1}-F_{2 k+2,2} \in L$. If $a=a_{2 k+1,2} \neq 0$ setting $y_{i}=x_{i}$ for $1 \leqq i \leqq 2 k+1$ and $y_{j}=x_{j}-a^{-1} a_{j 2} x_{2 k+1}$ for $j>2 k+1$ we see that $\operatorname{sp}\left(y_{1}, \cdots, y_{2 k}\right) \subseteq L$ and $A=F_{1,2 k+1}+F_{2 k+1,2} a$. Then $F_{21}(\text { ad } A)^{3}=\left(F_{1,2 k+1}-F_{2 k+1,2} a\right) 3 a \in L$ so $F_{1,2 k+1} \in L$. This contradicts Condition $\sim \mathrm{B}$ so we must have $a=0$. Also by Condition $\sim \mathrm{B}$ some $a_{i 2} \neq 0$. Hence we can choose $y_{2 k+2} \in\left\langle x_{2 k+2}, \cdots, x_{n}\right\rangle$ so that $y_{2 k+2} A=$ $-x_{2}$. Then setting $y_{i}=x_{i}$ for $1 \leqq i \leqq 2 k+1$ and choosing $y_{j}$ $\in\left\langle x_{2 k+2}, \cdots, x_{n}\right\rangle \cap \operatorname{ker} A$ for $j>2 k+2$ so that $\left\{y_{1}, \cdots, y_{n}\right\}$ is a basis for $V$ we have the result.

Now assume $\operatorname{sp}\left(x_{1}, \cdots, x_{2 k}\right) \subseteq L$ and $E_{1,2 k+1}-E_{2 k+2,2} \in L$. We will show that for some basis $\left\{y_{1}, \cdots, y_{n}\right\}$ of $V$ we have $\operatorname{sp}\left(y_{1}, \cdots, y_{2 k}\right)$ $\subseteq L$ and $F_{1,2 k+1}-F_{2 k+2,2}, F_{2 k+1,2 k+2} \in L$, thus proving (e). We have $E_{2 k+2,2 k+1}=E_{21}\left(\operatorname{ad}\left(E_{1,2 k+1}-E_{2 k+2,2}\right)\right)^{2} / 2 \in L$. Then as in (c) we have $B=\sum E_{i j} b_{i j} \in L$ where the $b_{i j} \in \Phi$ and satisfy $b_{2 k+1,2 k+2}=-1, b_{i j}$ $=-b_{i, 2 k+2} b_{2 k+1, j}$, and $\sum_{r} b_{i r} b_{r j}=0$ for $1 \leqq i, j \leqq n$. Then if $y_{2 k+2}$ $=-x_{2 k+1} B, y_{2 k+1}=x_{2 k+1}$, and $y_{j}=x_{j}+b_{j, 2 k+2} x_{2 k+1}$ for $j \neq 2 k+1,2 k+2$ we have $F_{2 k+1,2 k+2}=-B \in L, \quad F_{2 k+2,2 k+1}=E_{2 k+2,2 k+1} \in L$, and $F_{1,2 k+1}$ $-F_{2 k+2,2}=E_{1,2 k+1}-E_{2 k+2,2}-E_{2 k+2,2 k+1}\left(b_{2,2 k+2}-b_{2 k+1,1}\right) \in L$. Also, for 1 $\leqq i, j \leqq 2 k$, we have

$$
F_{i j}=E_{i j}-E_{i j}\left(\operatorname{ad} F_{2 k+1,2 k+2}\right)\left(\operatorname{ad} F_{2 k+2,2 k+1}\right)-F_{2 k+2,2 k+1} b_{j i} .
$$

Hence $\operatorname{sp}\left(y_{1}, \cdots, y_{2 k}\right) \subseteq L$ and $F_{1,2 k+1}-F_{2 k+2,2}, F_{2 k+1,2 k+2} \in L$ as required.

(f): If $\operatorname{dim} V=2 k$ and $\operatorname{sp}(V) \subseteq L$ then for $0 \leqq i \neq j \leqq k-1$ if $A \in L$ then

$$
\begin{aligned}
& E_{2 i+1,2 j+1} a_{2 i+1,2 j+1}+E_{2 j+2,2 i+2} a_{2 j+2,2 i+2} \\
& \quad=A\left(\operatorname{ad} E_{2 i+2,2 i+1}\right)\left(\operatorname{ad} E_{2 i+1,2 i+2}\right)\left(\operatorname{ad} E_{2 j+1,2 j+2}\right)\left(\operatorname{ad} E_{2 j+2,2 j+1}\right) \in L .
\end{aligned}
$$

Then by Condition $\sim \mathrm{B}$ we must have $a_{2 i+1,2 j+1}+a_{2 j+2,2 i+2}=0$. Similarly we see that $a_{2 i+1,2 j+2}=a_{2 j+1,2 i+2}$ and $a_{2 i+2,2 j+1}=a_{2 j+2,2 i+1}$. Hence $A=D+S$ where $S \in \operatorname{sp}(V)$ and $D=\operatorname{diag}\left\{d_{1}, \cdots, d_{2 k}\right\} \in L$. Now

$$
D\left(\operatorname{ad}\left(E_{1,2 i+1}-E_{2 i+2,2}\right)\right)=E_{1,2 i+1}\left(d_{1}-d_{2 i+1}\right)-E_{2 i+2,2}\left(d_{2 i+2}-d_{2}\right) \in L .
$$

Thus, again by Condition $\sim \mathrm{B}$, we have $d_{1}-d_{2 i+1}=d_{2 i+2}-d_{2}$ for all $0 \leqq i \leqq k-1$. Thus $2 D=I\left(d_{1}+d_{2}\right)+E$ where $E \in \operatorname{sp}(V)$. Thus $L$ $=\operatorname{sp}(V)$ or $L=\operatorname{csp}(V)$.

\section{REFERENCES}

1. E. Cartan, Les groupes de transformations continus, infinis, simples, Ann. Sci. École Norm. Sup. 26 (1909), 93-161; Oeuvres complètes. Vol. 2, Partie II, GauthierVillars, Paris, 1953, pp. 857-925. 
2. V. Guillemin, D. Quillen and S. Sternberg, The classification of the irreducible complex algebras of infinite type, J. Analyse Math. 18 (1967), 107-112. MR 36 \#221.

3. V. G. Kac, Simple irreducible graded Lie algebras of finite growth, Izv. Akad. Nauk SSSR Ser. Mat. 32 (1968), 1323-1367 = Math. USSR Izv. 2 (1968), 1271-1311.

4. S. Kobayashi and T. Nagano, On filtered Lie algebras and geometric structures. I, J. Math. Mech. 13 (1964), 875-907. MR 29 \#5961.

5. - On filtered Lie algebras and geometric structures. III, J. Math. Mech. 14 (1965), 679-706. MR 32 \#5803.

6. B. Kostant, A characterization of the classical groups, Duke Math. J. 25 (1958), 107-123. MR 20 \#2381.

7. A. I. Kostrikin, Simple Lie p-algebras, Trudy Mat. Inst. Steklov. 64 (1961), 79-89; English transl., Amer. Math. Soc. Transl. (2) 55 (1966), 195-206. MR 24 \#A1933.

8. G. B. Seligman, Modular Lie algebras, Ergebnisse der Mathematik und ihrer Grenzgebiete, vol. 40, Springer-Verlag, Berlin, 1967. MR 39 \#6933.

9. I. M. Singer and S. Sternberg, The infinite groups of Lie and Cartan. I. The transitive groups, J. Analyse Math. 15 (1965), 1-114. MR 36 \#911.

Courant Institute of Mathematical Sciences, New York University, New YORK, NEW YORK 10012 